\title{
Using forward markets to improve electricity market design ${ }^{\text {is }}$
}

\author{
Lawrence M. Ausubel, Peter Cramton* \\ University of Maryland, College Park, MD 20742, United States
}

\section{A R T I C L E I N F O}

Article history:

Received 9 January 2010

Received in revised form

25 May 2010

Accepted 26 May 2010

\section{Keywords:}

Electricity market design

Forward market

Capacity market

Firm energy market

Resource adequacy

\begin{abstract}
A B S T R A C T
Forward markets, both medium term and long term, complement the spot market for wholesale electricity. The forward markets reduce risk, mitigate market power, and coordinate new investment. In the medium term, a forward energy market lets suppliers and demanders lock in energy prices and quantities for one to three years. In the long term, a forward reliability market assures adequate resources are available when they are needed most. The forward markets reduce risk for both sides of the market, since they reduce the quantity of energy that trades at the more volatile spot price. Spot market power is mitigated by putting suppliers and demanders in a more balanced position at the time of the spot market. The markets also reduce transaction costs and improve liquidity and transparency. Recent innovations to the Colombia market illustrate the basic elements of the forward markets and their beneficial role.
\end{abstract}

(c) 2010 Elsevier Ltd. All rights reserved.

\section{Introduction}

Electricity market design seeks to satisfy consumers' demand for electricity at minimum cost. This requires both short-run efficiency - the operation of existing resources at minimum cost-and long-run efficiency-investment in the right quantity and mix of resources. Both goals are made difficult by features of electricity markets. Supply and demand must be balanced at every instant and at every location. The physical constraints of the network must be respected. And demand often is unresponsive to spot price fluctuations.

Our thesis is that the goals of electricity market design are better met when the spot market is complemented with two forward markets, one medium term and one long term. A well-designed spot market is necessary for efficiency, but is not sufficient. Efficiency requires that issues of risk, market power, and investment be addressed. The two forward markets address risk by enabling the market participants to lock in prices and quantities, limiting exposure to the more volatile spot market. Market power is addressed by

\footnotetext{
is Since 2006, Peter Cramton has advised the Colombian government on the design of long-term and medium-term forward markets for electricity; he advised ISO New England on the design of the Forward Capacity Market. Larry Ausubel has assisted the implementation of the Forward Capacity Market for ISO New England and has certified the first three Forward Capacity Auctions. This paper is based in part on this work. The paper also benefits from collaborations with Steven Stoft on forward reliability markets in both Colombia and New England. We are grateful to the National Science Foundation for funding.

* Corresponding author.

E-mail address: pcramton@gmail.com (P. Cramton).
}

putting participants in a more balanced position entering the spot market, mitigating the incentive to distort bids. Finally, the longterm market coordinates investment in new resources, assuring that adequate resources will be available when they are most needed.

The California electricity crisis of 2000-2001 illustrates all too well the problems that can arise when one relies excessively on the spot market. Key conditions of the crisis were insufficient forward contracting and tight supply. During this prolonged period of tight supply, the unhedged demanders were exposed to sustained high spot prices. Suppliers, also positioned without forward contracts, had strong incentives to exercise market power further exacerbating the high prices. The load serving entities, despite initially being well capitalized, ultimately teetered toward bankruptcy and the market collapsed.

The proposed forward markets would have reduced or perhaps prevented the crisis. To the extent that the crisis was caused by inadequate resources, the long-term market would assure sufficient investment to relieve the tight supply conditions that contributed to high prices. Even if spot prices became high for an extended period, with the forward markets the vast majority of the quantity would be transacted at sustainable forward prices, preventing the large wealth transfers that pushed utilities toward bankruptcy. Finally, suppliers in roughly balanced positions entering the spot market would have much reduced incentives to exercise market power, so the behavior of the spot market would have likely been less extreme.

Our view is that forward markets can address three of the pressing problems in current wholesale markets: investment, risk, and market power. 
The need for forward markets and the form that they take will depend on the setting. We use Colombia to illustrate one approach to the forward markets. Colombia recently adopted both a longterm market and a medium-term market. Similar markets have been adopted in parts of the U.S., such as New England. We present the essential features of each market and discuss some of the design choices. We begin with the long-term market and then discuss the medium-term market.

The need for regulated forward markets in electricity comes largely from market failures on the demand side. Consumer demand response is limited; consumers have limited exposure to spot prices and have no ability to express preferences for reliability. As a result, in most markets, regulators establish the quantity of resources needed. The long-term market assures that adequate resources exist and establishes a transparent competitive process for identifying and pricing these resources. Regulation of mediumterm (1-3 year) markets may be justified to avoid self-dealing by vertically integrated distribution companies, to address market power, and to reduce transaction costs.

\section{Long-term: the forward reliability market for investment}

Forward reliability markets now operate in Colombia and New England. These markets coordinate investment in new resources to assure that adequate resources are available when they are needed most. The two markets have the same basic features, although they differ in the definition of the reliability product. ${ }^{1}$

A fundamental characteristic of the Colombian electricity market is that it is hydro-dominated. Roughly $80 \%$ of Colombia's energy is produced from hydro resources, and about two-thirds of its capacity is hydro. As a result, the resource adequacy constraint in Colombia is having sufficient thermal resources and hydro reservoirs to provide energy during severe dry periods. Thus, Colombia has a "firm energy market" to assure resource adequacy, where the product (firm energy) is the ability to produce energy during severe dry periods.

By contrast, New England is a thermal-dominated system. The resource adequacy constraint is having sufficient resources to serve the annual peak. The reliability product is capacity-the ability to produce energy during a hot summer day. Thus, New England has a capacity market.

In both Colombia and New England, the regulator establishes the overall resource requirement and determines the individual contribution toward this requirement of each resource. For example, in Colombia each hydro unit (and thermal unit) is rated based on its expected energy output in a severe dry season. The system operator then procures on behalf of load enough firm energy to satisfy the requirement.

The first question is why have a forward reliability market. A main motivation is coordinating investment in new resources to assure resource adequacy. The absence of demand response is another factor. Spot prices tend to be too low during scarcity, either because of price caps or operator decisions, such as voltage reductions, which impact price. The forward reliability market reduces risk and addresses market power, while assuring that investment incentives are strong enough for efficient entry.

Issues of risk and investment incentives are especially important in Colombia. As a result of El Nino phenomena, sustained scarcity events from lack of rainfall occur about once every ten years. An investor is unlikely to build a thermal peaking unit anticipating the

\footnotetext{
${ }^{1}$ See Cramton and Stoft (2008) for a general analysis of forward reliability markets. Cramton and Stoft (2007) focus on the Colombia design; and Cramton (2006) focuses on the New England design.
}

energy profits during these severe dry periods. These events, occurring perhaps two months in ten years, are simply too infrequent. The investment would entail too much risk. The reliability market provides a constant revenue stream in exchange for the obligation to provide energy at more moderate prices during scarcity events. Risk is dramatically reduced for the investor (Cramton et al., 2006). Risk is also reduced for the demand side, which is important in Colombia where the demand side cannot tolerate large and sustained price shocks. Finally, demand is growing rapidly in Colombia, so new investment is essential.

Previous to the firm energy market, Colombia generators received a reliability payment, but this payment was set administratively. The new market selects new projects and establishes the payment through a transparent competitive process.

A main feature of the design is that the procurement occurs well in advance of the commitment period. This allows new projects to compete in advance of entry, before significant costs are sunk, which increases competition and produces a meaningful price. It also allows for coordinated entry, reducing the boom-and-bust cycles that frequently occur in the construction of new power plants.

The coordinated entry reduces the uncertainty in achieving the target level of resources. Some over-procurement will occur as a result of the lumpiness of investment and mistaken load forecasts, but it is not necessary to deliberately procure extra resources in recognition of uncertain entry.

A further advantage of forward procurement is that new resources set the firm energy price directly. Foreclosure of the market is prevented with limits on excessive concentration of supply.

With forward procurement, it is possible to make a long-term commitment for new resources. This reduces investor risk and sends a price signal for new investment that is directly related to the cost of new entry.

The firm energy product is a financial call option backed by a physical resource rated by the regulator as capable of producing a particular level of energy during a severe dry period. The physical requirement guarantees that sufficient resources will be available to produce energy. The financial call option hedges load from highenergy prices during periods of scarcity. ${ }^{2}$

Bundling the physical resource to the product is essential. This assures adequate physical resources and enables the market to restore the "missing money" in the energy market, caused by toolow energy prices as a result of prices caps or operator actions during scarcity. Because of this missing money, the clearing price in the firm energy market exceeds the financial cost of the call option.

The supplier's generating units and fuel provide a physical hedge to limit the risk of selling the call option. Indeed, relative to an energy-only market, the supplier's risk is reduced, since the firm energy market substitutes highly variable energy rents with a constant firm energy payment.

The supplier's obligation is load following in aggregate: in each hour the total obligation is equal to load. A supplier's obligation in any day is equal to its share of firm energy. The obligation is distributed over the day based on the supplier's hourly dispatch. This definition-tying a unit's obligation to its hourly dispatch during scarcity-reduces supplier deviations. A baseload unit's obligation is spread throughout the day; a hydro unit with high opportunity cost has its obligation concentrated on the peak hours of the day.

\footnotetext{
${ }^{2}$ Many papers have suggested the use of call options. See Bidwell (2005), Vazquez et al. (2002), Chao and Wilson (2004), Cramton and Stoft (2006, 2007, 2008), and Oren (2005).
} 
A desirable variation is to base the obligation on expected load, rather than actual load. This reduces supplier risk and improves incentives for demand response.

The strike price of the call option, called the scarcity price, is set at a high heat-rate times a gas index plus other non-fuel variable costs. The intent is for the scarcity price to be above the marginal cost of all thermal units. Although a lower scarcity price may further reduce price risk, the scarcity price is set high to allow energy contracting outside of the long-term market, for example in bilateral contracts or the medium-term market described in the next section.

The auction is conducted four or more years in advance of the commitment period. New resources lock in a firm energy price for up to twenty years. ${ }^{3}$ Existing resources receive the price set by new entry each year. All resources, both new and existing, are paid the same firm energy price. Moreover, this price is the same for all locations and for all types of resources. In markets with binding transmission constraints, the reliability market may have zonal prices that reflect the differing value of resources in different zones, and the price is then the same for all types of resources at all locations within a given zone.

The auction uses a descending-clock format. ${ }^{4}$ The price starts at a high price, about twice the estimated cost of new entry. Suppliers respond with the quantity each is willing to supply at that price. If there is excess supply, the price is reduced and the suppliers again specify the quantity desired at the lower price. This process continues until a price is reached in which supply and demand are balanced. This defines the clearing price, which is paid to all winning bidders throughout their commitment periods.

The quantity of firm energy demanded increases slightly as the price falls. Additional firm energy beyond the target has value for consumers, but this marginal value declines fairly rapidly. The demand curve has both a price ceiling and a price floor. The price ceiling at two times the estimated cost of new entry (CONE) reflects the fact that entry at high prices is not limited by the price incentive, but rather other non-price constraints. The price floor at onehalf CONE is intended to prevent the firm energy price from falling too low in times of surplus. This provides stability to the firm energy price, which reduces supplier risk and thus reduces consumer cost in the long term.

The descending-clock auction includes important price formation features. First, to promote price discovery, there is an activity rule that requires each supplier's offers to be consistent with an upward-sloping supply curve; that is, as the price declines a supplier can only maintain or decrease its supply. Second, to prevent the exercise of market power by existing suppliers, existing supply can opt out of the market, but this choice is not allowed to impact the price paid to existing suppliers.

Performance incentives are provided primarily via the spot market. To the extent that the supplier provides more than its obligation, it is rewarded by the spot energy price; to the extent that it supplies less than its obligation, it loses the energy price, just as in a contract for differences. Hence, the supplier has the same marginal incentives as if it had not sold a hedge. If the supplier is able to shift its output to higher-priced hours, it will be rewarded. Because such shifting is rewarded, the option has no effect on

\footnotetext{
${ }^{3}$ In New England, the auction is conducted approximately three years in advance of the commitment period and new resources lock in a capacity price for up to five years.

${ }^{4}$ See Ausubel and Cramton (2004) for a general analysis of clock auctions and Ausubel and Cramton (in this issue) for a more detailed discussion of ascending-
} clock auctions in electricity markets. performance incentives, but acts only to hedge load and suppliers. Performance incentives come from the spot market, as they should, and are not affected by the hedge.

An advantage of the hedge is that it reduces the incentive to exercise market power during times of scarcity. This is because suppliers have effectively sold forward energy covering $100 \%$ of load during times of scarcity. The forward sale improves the performance of the spot energy market during scarcity periods, which is exactly when the market is most vulnerable to market power. Additionally, the hedge reduces supplier risk by removing the energy rents above the scarcity price, which tend to have high variance, and by including them in the firm energy payment, which does not vary with weather or other factors outside of the supplier's control.

The auction design includes fail-safe mechanisms that determine what happens in the unlikely event that there is inadequate supply offered in the auction, or there is insufficient competition.

The auction design facilitates an active secondary market for the firm energy product. Although the primary auction is intended to procure all or nearly all of the target quantity, the purchase occurs several years in advance and circumstances may change. Thus, on an annual basis reconfiguration auctions are held as needed, so that suppliers and demanders can balance their positions for each commitment year that has yet to occur. The reconfiguration auction is a sealed-bid clearing price auction. Sellers submit offers and buyers submit bids, and a uniform clearing price is determined.

Every electricity market requires sufficient capacity and energy to reliably satisfy load. In Colombia, we focus on firm energy-the ability to generate electricity in dry periods-because that is the reliability constraint that is currently binding in Colombia. Today there is roughly $13 \mathrm{GW}$ of capacity to service the annual peak of about $8 \mathrm{GW}$. There is a $5 \mathrm{GW}$ or $62 \%$ surplus of capacity. Given this surplus, it is clear that the market price for capacity is zero. Firm energy - the ability to generate energy in severe dry periods-is the scarce resource, and that is what consumers should pay for. By contrast, in the electricity markets in the thermal-dominated U.S. markets, the reliability constraint is having enough capacity to handle the annual peak, so the focus there is on capacity.

In some markets it may be unclear whether capacity or firm energy is the scarce resource. The auction can accommodate scarcity of both firm energy and capacity. The extension is simple. The only change is introducing a second product, capacity. As before suppliers offer resources and each resource is a package of both firm energy and capacity. The market identifies the collection of resources that satisfy both the firm energy and capacity constraints at minimum cost. This is done in the descending-clock auction as before, but now there are two products and so two prices. In each round, the auctioneer names a pair of prices $\left(\mathrm{p}_{\mathrm{E}}, \mathrm{p}_{\mathrm{C}}\right)$, a price for firm energy and a price for capacity. Each supplier then decides whether, given the pair of prices, the supplier wishes to offer its resource. The auctioneer then determines the aggregate supply of both firm energy and capacity. If there is excess supply of a product, the auctioneer reduces its price, unless the price is already zero. This process continues until there is no longer excess supply of any product with a positive price. The auctioneer's goal in adjusting prices is to find the market equilibrium where supply and demand balance for all products with positive prices.

\section{Medium-term: forward energy market}

We now turn to the forward energy market, which is a mediumterm market in which demanders and suppliers can lock in energy prices and quantities for one to three years. Again we focus on the forward energy market recently adopted in Colombia (Cramton, 2007). 
As in most electricity markets, the vast majority of Colombia's energy is settled according to energy contracts with terms that are much longer than the hourly spot market. Energy contracts often have durations of one or two years, and sometimes more. These energy contracts benefit both suppliers and demanders. Both sides of the market are able to lock in a price, and thereby reduce price risk from the more volatile spot market.

Unfortunately, the existing energy contracting market has high transaction costs, as a result of non-standard contracts, poor price formation, localized contracting, lack of transparency, and other factors. Evidence of a problem is seen in the frequent occurrence of higher contract prices for regulated customers compared with nonregulated customers, which is unexplained by load shapes, credit risks, and other factors. The forward energy market, which begins in 2010, is intended to address the existing problems in the informal bilateral contracting market.

The forward energy market is an organized market to procure energy for electricity customers on a forward basis. It includes the regulated customers (residential and other small customers) and optionally the nonregulated (large) customers. Currently, regulated customers represent $68 \%$ of the total electricity demand and nonregulated customers represent the remaining $32 \%$. The design is novel in allowing nonregulated customers to participate. Although the regulated and nonregulated energy products remain distinct, their integration into a single market facilitates arbitrage between the products, improves liquidity, and reduces transaction costs. Both regulated and nonregulated customers benefit from this unified approach.

Regulated customers are small customers without hourly meters; nonregulated customers are large customers with hourly meters. The nonregulated product makes use of hourly meters to encourage demand response. In addition, due to their large size, nonregulated customers are active buyers in the forward energy market, submitting demand bids. In contrast, the regulated customers have a more limited demand response capability and are not active buyers in the forward energy auction - their demands are set administratively.

The market is based on two products, a regulated product and a nonregulated product. Both products are standard financial contracts: take-or-pay (fixed quantity) energy contracts for $1 \mathrm{MWh}$ per day and have a commitment period of one calendar year. The nonregulated product has a flat load shape. The regulated product has the shape of historical regulated load, appropriately discounted for Saturdays (10\%) and Sundays and holidays (15\%). For the regulated product, each supplier bids to serve its desired quantity of Colombia's regulated load. The supplier is paid the clearing price for every MWh of energy supplied. Deviations between the supplier's hourly supply and obligation are settled at the spot energy price or the scarcity price, whichever is lower. The spot settlement price is capped at the scarcity price, since the firm energy market provides price coverage for prices above the scarcity price (about $\$ 260 / \mathrm{kWh}$ in January 2007 Colombian pesos; or US\$120/MWh); see Cramton and Stoft (2007).

The distribution companies purchase one-hundred percent of the expected regulated load on behalf of the regulated customers in a sequence of auctions. Thus, the forward energy market together with the firm energy market provides nearly $100 \%$ price coverage for the expected load of regulated customers. The forward energy market provides price coverage from zero to the scarcity price, and the firm energy market provides price coverage above the scarcity price. This accomplishes two things: 1) it provides rate stability for regulated customers, and 2) it provides revenue stability for suppliers. The result is reduced risk for both sides of the market. Price risk only remains to the extent actual demand differs from expected demand.

The goal is to have a regulated load shape (and discounts for Saturday, Sunday, and holidays) such that the purchase of the target quantity results in the smallest cumulative deviations over the year between actual demand and contracted demand for each hour over the year. This will provide the best hedge from volatile spot prices. For countries with substantial load variation due to weather or season, the regulated product would need to account for season and weather to better match actual load.

Basing the product on expected demand, rather than actual demand, has an important advantage going forward as real-time meters become available at the consumer level. The distribution company can include consumer contracts that hedge expected load, but reward the consumer for curtailing demand when spot prices are high. With the take-or-pay contracts the distribution company, and potentially the consumer, is exposed to the spot price on the margin. This also shifts the burden of estimating demand to the distribution company. Demand estimation should improve; reduced errors in demand forecasts by the distribution company will result in reduced exposure to spot prices.

The nonregulated product is a compromise emphasizing simplicity. It has a potential downside in that it does not make any attempt to follow the load shape of nonregulated customers. As a result, the product is an imperfect hedge, which may create market power problems in the spot market. If customers purchased a quantity sufficient to cover expected demand over the year, then customers would predictably find themselves with a spot surplus in off-peak periods and a spot deficit in peak periods. In the spot market, the customers would be selling when the price is low and buying when the price is high, resulting in positive deviation payments. In contrast, suppliers would be buying when the price is low (off-peak) and selling when the price is high (peak). Suppliers would have strengthened incentives to increase the within day variation in prices through the exercise of market power in the spot market.

Customers with variable demands will find the flat demand product less attractive. These customers may find the flat product is an inadequate hedge, and so may contract separately for load following (take-and-pay) contracts. Still the flat product provides a partial hedge and remains a possibility for all nonregulated customers.

From a supplier's point of view, the flat demand is attractive, especially for suppliers with less flexible resources. Suppliers with more flexible resources still may bid aggressively for the nonregulated product, anticipating the positive deviation payments they are apt to receive by shifting output to the peak hours.

Accommodating different load shapes for nonregulated customers would make the market much more complicated. The flat product is an attractive compromise provided market power concerns are not too important and provided nonregulated load is sufficiently flat that the product is attractive to most nonregulated customers.

Since the nonregulated market is voluntary, customers do have the option of limiting purchases of the flat product if they find it unattractive. In addition to bilateral forward contracts, customers can also use demand management to protect against supplier market power in the spot market.

The prior approach of bilateral contracts suffers from three problems. Price risk is greater since the contract cover often is incomplete. Market power in both the spot market and the bilateral market is more of a concern, since supplier positions are more apt to be out of balance entering the spot market and competition for bilaterals is weaker with specialized local products. ${ }^{5}$ The absence of

\footnotetext{
5 The greater transparency of the forward market facilitates the monitoring of large suppliers who may attempt to acquire an imbalanced position to exercise market power (Herrera Dappe, 2008).
} 
a standard contract also results in high transaction costs in addition to weaker competition. By contrast, the new market provides nearly full price coverage, suppliers likely enter the spot market with more balanced positions, and the single product minimizes transaction costs. In addition, the problem of self-dealing between the load serving entity and its supplier affiliate is eliminated.

The market is mandatory for regulated customers, but voluntary for suppliers. Mandatory participation on the demand side motivates robust participation on the supply side.

The nonregulated customers enjoy similar benefits as the regulated customers from the forward energy market. The main difference is that the nonregulated customers actively bid in the forward energy market, and thus decide how much contract cover to purchase and in which auction. The voluntary participation of nonregulated customers provides a useful competitive check in the market. If a nonregulated customer prefers different contracts, the customer can contract separately. Also nonregulated customers can arbitrage between the forward and spot energy markets.

The nonregulated product promotes rate stability. The product provides a substantial hedge, yet still exposes the customer to the spot price on the margin, motivating demand response.

There are a number of possible choices for the timing and frequency of auctions, and the duration of contracts. These three elements can be adjusted to manage price and credit risk, while minimizing transaction costs. Ultimately, Colombia decided on a calendar-year product purchased in a sequence of four to six auctions conducted one to two years ahead. The approach is simple and yet provides good time diversification, shielding customers from transient events. One-quarter to one-sixth of regulated load is purchased in each auction. At any one time, the customer rate reflects the average of four to six auctions equally spaced over a one-year period, one to two years ahead. ${ }^{6}$ Even the auction with the shortest planning period occurs more than twelve months before the start of the contract. This means that the auction price will be set before there is much resolution of how severe conditions will be in the dry season of the commitment year, reducing risk and improving rate stability. Also with a one-year contract, the cost of guarantees to assure performance are less.

The proposed forward energy market complements the other key elements of the Colombian market: the spot energy market and the firm energy market. Combined, the forward energy market and the firm energy market provide substantial price coverage. Not only does this reduce risk for both sides of the market, it puts suppliers in a more balanced position in the spot energy market. As a result, incentives to exercise market power are greatly mitigated in the spot market. This improves the price signal in the spot market, since a supplier in a balanced position has an incentive to offer its true marginal cost.

Efficient price formation is one of the most important objectives of the forward energy market. The simultaneous descending-clock auction is well suited to promote efficient price formation. The descending-clock auction provides excellent price discovery and enables suppliers to freely arbitrage across the regulated and nonregulated products. This assures that any price difference between the two products is a reflection of cost differences.

With just two products, there is a simple and powerful method that allows suppliers to express any linear substitution between the regulated and the nonregulated products (Cramton, 2007). This is desirable, since the products are close substitutes. In the descending-clock auction, each supplier expresses the total

\footnotetext{
${ }^{6}$ When supply is procured over a sequence of auctions, some monitoring of the market by the regulator is important to assure that large suppliers do not bid to enhance market power in the sequence of auctions (Herrera Dappe, 2009).
}

quantity it wishes to supply (both regulated and nonregulated) at a particular regulated price. The supplier also bids two price spreads. The higher price spread indicates the price spread (regulated price minus nonregulated price) above which the supplier supplies only nonregulated product; the lower price spread indicates the price spread below which the supplier supplies only regulated product; for intermediate spreads, the supplier is assumed to supply both products in proportion to how close the price spread is to the lower or upper price spreads. Each round the price spread is set to equalize excess supply in percentage terms across the two products.

The integration of the regulated and nonregulated markets can be expected to lead to greater liquidity, improved price formation, and lower transaction costs. Our view is that the forward energy market as proposed here will dramatically improve the energy contract market for both regulated and nonregulated customers, and improve the spot market as well, since suppliers typically will enter the spot market with a more balanced position, reducing incentives to exercise market power.

Since participation for nonregulated customers is voluntary, the organized forward market faces important competition from the bilateral market.

\section{Conclusion}

Forward markets can greatly improve the performance of wholesale electricity markets by addressing the critical problems of risk, market power, and investment. Colombia recently adopted both a long-term investment market (the firm energy market) and a medium-term market for forward energy.

Early capacity markets suffered from serious flaws that ultimately led to their elimination or replacement. The firm energy market design presented here corrects each of the principal flaws of earlier capacity markets. In particular:

1. The product is defined as a physically-backed call option on energy. The physical requirement assures adequate resources. The call option improves the performance of the spot energy market in times of scarcity and reduces supplier risk.

2. New entry is coordinated by holding the auction well in advance of the commitment period. This mitigates the boom-bust cycle that is common in electricity markets. More importantly, it allows the firm energy price to be more directly tied to the cost of new entry.

3. Strong performance incentives are maintained from the spot energy price. Additional incentives come from the link, especially for thermal resources, between historical performance and the level of firm energy certification.

4. Price formation is supported by minimizing the ability of existing suppliers to exercise market power in the firm energy market. In addition, the descending-clock auction format encourages price discovery and improves the efficiency of the auction outcome.

A successful firm energy market depends on more than good design. First, the firm energy market has at its foundation the spot energy market. It is important that the spot energy market send reliable price signals. Second, the firm energy market relies on competitive entry; hence, entry barriers must be kept to a minimum. And finally, the firm energy market depends on longrun price expectations; thus, it is important that investors have faith in the stability of the market over the long run.

Colombia's forward energy market promises to reduce transaction costs and enhance competition for regulated and nonregulated customers. 
The market is based upon two financial products, one for regulated customers and one for nonregulated customers. Suppliers bid to supply a quantity of the regulated load and a quantity of the nonregulated load-aggregated across all load serving entities. This simple approach enhances liquidity and competition, since all suppliers are competing for and trading in the same two products.

Since regulated customers are procuring $100 \%$ of the expected regulated load, these customers are nearly fully-hedged from the spot price. Similarly, suppliers are able to lock in a long-term price to stabilize their revenues.

Including nonregulated customers in the centralized market has two important advantages. First, substitution between the regulated and nonregulated products assures that both customer classes pay market-based rates for electricity. And second, the demand response of nonregulated customers in the forward energy market helps protect both nonregulated and regulated customers from supplier market power, and reduces the importance of the regulated demand curve in determining prices. Competition among suppliers and the competitive choices of the nonregulated customers determine the auction clearing prices.

The forward energy products are fully consistent with, and indeed complementary to, the other key elements in the Colombian market: the spot energy market and the firm energy market. The firm energy market together with the forward energy market put suppliers in a more balanced position in the spot market. Not only does this reduce risk for both sides of the market, it greatly mitigates incentives to exercise market power in the spot market. Thus, we anticipate that the forward energy market will not only reduce problems in the bilateral contracting market, but will improve the performance of the spot energy market. Both the electricity industry and its customers will benefit from these markets.

Efficient price formation is one of the most important objectives of the forward energy market. The simultaneous descending-clock auction is well suited to generate efficient price formation. The descending-clock auction provides excellent price discovery and enables suppliers to freely arbitrage across the regulated and nonregulated products. This assures that any price difference between the two products is a reflection of cost differences.
Our view is that Colombia's two forward markets support both the short-run and long-run efficiency goals of the wholesale market. The forward markets effectively address the key problems of risk, market power, and investment.

Some may view these forward markets as excessive central planning. We contend that the alternative of incomplete markets may be more dangerous, at least while demand-side market failures remain severe. The California electricity crisis of 2000-2001 demonstrates the importance of addressing risk, market power, and investment. The global financial crisis of 2008-2009 demonstrates the importance of doing so with transparent markets that trade economically-sensible products.

\section{References}

Ausubel, Lawrence M., Cramton, Peter, 2004. Auctioning many divisible goods. Journal of the European Economic Association 2, 480-493. April-May.

Ausubel, Lawrence M. and Peter Cramton. "Virtual power plant auctions," Utilities Policy, in this issue.

Bidwell, Miles, 2005. Reliability options. Electricity Journal June.

Chao, Hung-po, Wilson, Robert, 2004. Resource Adequacy and Market Power Mitigation via Option Contracts. EPRI, Palo Alto, CA.

Cramton, Peter, 2006. New England's Forward Capacity Auction. Working Paper. University of Maryland.

Cramton, Peter, 2007. Colombia's Forward Energy Market. Working Paper. University of Maryland.

Cramton, Peter, Stoft, Steven, 2006. The Convergence of Market Designs for Adequate Generating Capacity. white paper for the. California Electricity Oversight Board. March 2006.

Cramton, Peter and Steven Stoft (2007), “Colombia firm energy market," Proceedings of the Hawaii International Conference on System Sciences, vol. 40.

Cramton, Peter, Stoft, Steven, 2008. Forward reliability markets: less risk, less market power, more efficiency. Utilities Policy 16, 194-201.

Cramton, Peter, Stoft, Steven, West, Jeffrey, 2006. Simulation of the Colombian Firm Energy Market. Working Paper. University of Maryland.

Herrera Dappe, Matias, 2008. Market Power, Forward Trading, and Supply Competition. Working Paper. University of Maryland.

HerreraDappe, Matias, 2009. Sequential Uniform-Price Auctions. Working Paper. University of Maryland.

Oren, Shmuel S, 2005. Generation adequacy via call option obligations: safe passage to the promised land. Electricity Journal November.

Vazquez, Carlos, River, Michel, Arriaga, Ignacio Perez, 2002. A market approach to long-term security of supply. IEEE Transactions on Power Systems 17 (2), 349-357. 